\title{
FROM ENDOMORPHISMS TO AUTOMORPHISMS AND BACK: DILATIONS AND FULL CORNERS
}

\author{
MARCELO LACA
}

\begin{abstract}
When $S$ is a discrete subsemigroup of a discrete group $G$ such that $G=$ $S^{-1} S$, it is possible to extend circle-valued multipliers from $S$ to $G$; to dilate (projective) isometric representations of $S$ to (projective) unitary representations of $G$; and to dilate/extend actions of $S$ by injective endomorphisms of a $\mathrm{C}^{*}$-algebra to actions of $G$ by automorphisms of a larger $\mathrm{C}^{*}$-algebra. These dilations are unique provided they satisfy a minimality condition. The (twisted) semigroup crossed product corresponding to an action of $S$ is isomorphic to a full corner in the (twisted) crossed product by the dilated action of $G$. This shows that crossed products by semigroup actions are Morita equivalent to crossed products by group actions, making powerful tools available to study their ideal structure and representation theory. The dilation of the system giving the BostConnes Hecke $\mathrm{C}^{*}$-algebra from number theory is constructed explicitly as an application: it is the crossed product $C_{0}\left(\mathbb{A}_{f}\right) \rtimes \mathbb{Q}_{+}^{*}$, corresponding to the multiplicative action of the positive rationals on the additive group $\mathbb{A}_{f}$ of finite adeles.
\end{abstract}

\section{INTRODUCTION}

In recent years there has been renewed interest in crossed products by semigroups of endomorphisms, viewed now as universal algebras in contrast to their original presentation as corners in crossed products by groups. This new approach, initiated by Stacey [31] following a strategy pioneered by Raeburn for crossed products by group actions [30], is based on the explicit formulation of a semigroup crossed product as the universal $\mathrm{C}^{*}$-algebra of a covariance relation. As such, it motivated the development of specific techniques and brought about new insights and applications, e.g. [31, 2, 25, 11, 17, 20, 21, 3, 18]. Nevertheless, the implicit view of semigroup crossed products as corners continues to have a very important role: it is often invoked to prove the existence of nontrivial universal objects and it allows one to import results from the well-developed theory of crossed products by groups. When the endomorphisms are injective and the semigroup is abelian the two approaches are equivalent, and the proof involves using a direct limit to transform the endomorphisms into automorphisms and the isometries into unitaries. This has been done when the abelian semigroup is $\mathbb{N}$ [11, 31], when it is totally ordered [1], and, in general, when it is cancellative [25]. As crossed products by more general (nonabelian) semigroups are being considered from the universal property point of view, the need arises to determine whether a realization as corners in crossed products by groups is true and useful in those cases too. This is the main task undertaken in the present work.

A step away from commutativity of the acting semigroup was taken in [19] where isometric representations and multipliers of normal cancellative semigroups were extended using the same direct limits (the semigroup $S$ is normal if $x S=S x$ for every $x \in S$, in which case the natural notions of right and left orders on $S$ coincide). Here we will go further and consider discrete semigroups that can be embedded in a discrete group and for which the right order is cofinal; since cofinality is a key ingredient of a directed system,

Date: January 25, 1998; revised October 12, 1998.

Research supported by the Australian Research Council.

Typeset by $\mathcal{A M}_{\mathcal{M}}$-LATEX 
this class is, arguably, the most general one for which the usual direct limit construction would work without a major modification.

Based on the results presented below one may argue that the relevant object is the action of an ordered group, and that there are two ways of looking at it; the first is as an automorphic action on a $\mathrm{C}^{*}$-algebra taken together with a distinguished subalgebra which is invariant under the action of the positive cone, and the second is simply as the endomorphic action of this positive cone on the invariant subalgebra. We show that these two points of view are equivalent: to go from the former to the latter one just cuts down the automorphisms to endomorphisms of the invariant subalgebra and restricts to the positive cone, and the process is reversed by way of a dilation-extension construction, Theorem 2.1.1, which constitutes our first main result. We also explicitly state and prove two additional features of this equivalence that, in our opinion, have not previously received enough attention. The first one is that the minimal automorphic dilation is canonically unique, which for instance allows one to test a good candidate, as done in Subsection 3.2 below. The second one is that the crossed product by the semigroup action is realized as a full corner in the crossed product by a group action, so the equivalence of the two approaches technically translates into the strong Morita equivalence of the crossed products. This is done in Theorem 2.2.1, which is our second main result.

A modicum of extra work shows that these results are also valid for twisted crossed products and projective isometric representations with circle-valued multipliers. This requires the easy generalization, to Ore semigroups, of results known for semigroups that are abelian [4, 12, 8, 16] or normal [19, 26], which is done in the preliminary subsections 1.2 and 1.3. The arguments given are for projective isometric representations and twisted crossed products, but setting all multipliers to be identically 1 will lighten the burden slightly for those interested in the dilation-extension itself and not in projective representations, twisted crossed products, and extensions of multipliers.

In the final section we give an application to the semigroup dynamical system from number theory [21] which has the Bost-Connes Hecke $\mathrm{C}^{*}$-algebra [5] as its crossed product. Starting with the $p$-adic version of the system [18, Section 5.4] we show how one is quite naturally led to consider the ring of finite adeles with the multiplicative action of the positive rationals. This establishes a natural heuristic link between the Bost-Connes Hecke $\mathrm{C}^{*}$-algebra and the space $\mathcal{A} / \mathbb{Q}^{*}$, which lies at the heart of Connes's recent formulation of the Riemann Hypothesis as a trace formula [9, 10].

\section{Preliminaries}

In this first section we gather the basic definitions and results concerning the semigroups on which we will be interested. We also generalize other results about isometries and crossed products that are valid, with more or less the same proofs, in the present setting, although they were originally stated for particular cases.

\subsection{Ore semigroups.}

Definition 1.1.1. An Ore semigroup $S$ is a cancellative semigroup such that $S s \cap S t \neq \emptyset$ for every pair $s, t \in S$. Ore semigroups are also known as right-reversible semigroups. (We leave the obvious symmetric consideration of left-reversibility to the reader.)

Theorem 1.1.2 (Ore, Dubreil). A semigroup $S$ can be embedded in a group $G$ with $S^{-1} S=$ $G$ if and only if it is an Ore semigroup. In this case, the group $G$ is determined up to canonical isomorphism and every semigroup homomorphism $\phi$ from $S$ into a group $\mathcal{G}$ extends uniquely to a group homomorphism $\varphi: G \rightarrow \mathcal{G}$. 
Proof. See e.g. theorems 1.23, 1.24 and 1.25 in []] for the first part. We only need to prove the assertion about extending $\phi$. Since $G=S^{-1} S$, given $x, y \in S$ there exist $u, v \in S$ such that $v^{-1} u=y x^{-1}$, and hence the element $u x=v y$ is in $S x \cap S y$, proving that $S$ is directed by the relation defined by $s \preceq_{r} t$ if $t \in S s$. An easy argument shows that $\varphi\left(x^{-1} y\right)=\phi(x)^{-1} \phi(y)$ defines a group homomorphism from $G=S^{-1} S$ to $\mathcal{G}$ that extends $\phi$.

Remark 1.1.3. The last assertion of the theorem generalizes [19, Lemma 1.1]. Here we have found it more convenient, for compatibility with the rest of [19], to work with the right order $\preceq_{r}$ determined by $S$ on $G$ via $x \preceq_{r} y$ if $y \in S x$.

To illustrate the class of semigroups being considered, we list a few examples which have appeared recently in the context of semigroup actions:

- Abelian semigroups, (notably the multiplicative nonzero integers in an algebraic number field [3]);

- Semigroups obtained by pulling back the positive cone from a totally ordered quotient [29];

- Normal semigroups, in particular semidirect products [19, 26];

- Groups of matrices over the integers having positive determinant [6, Example 4.3];

1.2. Extending multipliers and dilating isometries. Let $\lambda$ be a circle-valued multiplier on $S$, that is, a function $\lambda: S \times S \rightarrow \mathbb{T}$ such that

$$
\lambda(r, s) \lambda(r s, t)=\lambda(r, s t) \lambda(s, t), \quad r, s, t \in S .
$$

A projective isometric representation of $S$ with multiplier $\lambda$ on a Hilbert space $H$ (an isometric $\lambda$-representation of $S$ on $H$ ) is a family $\left\{V_{s}: s \in S\right\}$ of isometries on $H$ such that $V_{s} V_{t}=\lambda(s, t) V_{s t}$.

A twisted version of Ito's dilation theorem [14] was obtained in [19, Theorem 2.1], where projective isometric representations of normal semigroups were dilated to projective unitary representations. Essentially the same proof, inspired on Douglas's [13], works for Ore semigroups and gives the following.

Theorem 1.2.1. Suppose $S$ is an Ore semigroup and let $\left\{V_{s}: s \in S\right\}$ be an isometric $\lambda$-representation of $S$ on a Hilbert space $H$, where $\lambda$ is a multiplier on $S$. Then there exists a unitary $\lambda$-representation of $S$ on a Hilbert space $\mathcal{H}$ containing a copy of $H$ such that

(i) $U_{s}$ leaves $H$ invariant and $\left.U_{s}\right|_{H}=V_{s}$; and

(ii) $\bigcup_{s \in S} U_{s}^{*} H$ is dense in $\mathcal{H}$.

Proof. Verbatim from the proof of [19, Theorem 2.1], except for the following minor modification of the part of the argument where normality is used to obtain an admissible value for the function $f_{t}$. The value st used there has to be substituted by any (fixed) $z \in S s \cap S t$, and thus the fourth paragraph there should be replaced by the following one.

Suppose now that $f \in H_{0}$ and $t \in S$, and consider the function $f_{t}$ defined by $f_{t}(x)=$ $\lambda(x, t) f(x t)$ for $x \in S$. If $s \in S$ is admissible for $f$, let $z \in S s \cap S t$. We will show that $s_{0}:=z t^{-1}$ is admissible for $f_{t}$. For every $x \in S s_{0}, x t \in S z$, and since $z$ is admissible for $f$

$$
\begin{aligned}
\lambda(x, t) f(x t) & =\lambda(x, t) \overline{\lambda\left(x t z^{-1}, z\right)} V_{x t z^{-1}} f(z) \\
& =\overline{\lambda\left(x t z^{-1}, z t^{-1}\right)} V_{x t z^{-1}} \lambda\left(z t^{-1}, t\right) f\left(z t^{-1} t\right) \\
& =\overline{\lambda\left(x s_{0}^{-1}, s_{0}\right)} V_{x s_{0}^{-1}} f_{t}\left(s_{0}\right)
\end{aligned}
$$


where the second equality holds by the multiplier property applied to the elements $x t z^{-1}$, $z t^{-1}$, and $t$ in $S$. This proves that $s_{0}$ is admissible for $f_{t}$, so $f_{t} \in H_{0}$.

Since the results of [19] concerning discrete normal semigroups depend only on this dilation theorem and on the unique extension of group-valued homomorphisms, they too are valid for Ore semigroups and we list them here for reference.

Theorem 1.2.2. Suppose $S$ is an Ore semigroup and let $G=S^{-1} S$. Then

1. Every multiplier on $S$ extends to a multiplier on $G$.

2. Restriction of multipliers on $G$ to multipliers on $S$ gives an isomorphism of $H^{2}(G, \mathbb{T})$ onto $H^{2}(S, \mathbb{T})$.

3. Suppose $\lambda$ is a multiplier on $S$ and let $V$ be a $\lambda$-representation of $S$ by isometries on $H$. Assume $\mu$ is a multiplier on $G$ extending $\lambda$. Then there exists a unitary $\mu$-representation $U$ of $G$ on a Hilbert space $\mathcal{H}$ containing a copy of $H$ such that $U_{s} \mid H=V_{s}$ for $s \in S$, and $\bigcup_{s \in S} U_{s}^{*} H$ dense in $\mathcal{H}$. Moreover, $U$ and $\mathcal{H}$ are unique up to canonical isomorphism.

Proof. The proofs of all but the last statement about uniqueness are as in Theorem 2.2, Corollary 2.3 and Corollary 2.4 of [19], provided one considers the left-quotients $x=t^{-1} s$ instead of the right-quotients used there. In order to prove the uniqueness statement suppose $\left(U^{\prime}, \mathcal{H}^{\prime}\right)$ is another unitary $\mu$-representation such that $U_{s}^{\prime} \mid H=V_{s}$ and $\bigcup_{s \in S} U_{s}^{\prime *} H$ is dense in $\mathcal{H}^{\prime}$. It is easy to see that the map

$$
W: U_{s}^{*} h \mapsto U_{s}^{\prime *} h, \quad s \in S, h \in H
$$

is isometric, and that it extends to an isomorphism of $\mathcal{H}$ to $\mathcal{H}^{\prime}$ because of the density condition. It only remains to show that $W$ intertwines $U$ and $U^{\prime}$. Since $S$ is an Ore semigroup, for every $x$ and $s$ in $S$ there exist $z$ and $t$ in $S$ such that $x s^{-1}=t^{-1} z$. Then $t x=z s$, so

$$
\begin{aligned}
W U_{x}\left(U_{s}^{*} h\right) & =W U_{x} U_{t x}^{*} U_{z s} U_{s}^{*} h=\mu(t, x) \overline{\mu(z, s)} W U_{t}^{*} U_{z} h=\mu(t, x) \overline{\mu(z, s)} W U_{t}^{*}\left(V_{z} h\right) \\
& =\mu(t, x) \overline{\mu(z, s)} U_{t}^{\prime *}\left(V_{z} h\right)=\mu(t, x) \overline{\mu(z, s)} U^{\prime *}{ }_{t}^{\prime} U_{z}^{\prime} h=U^{\prime}{ }_{x}\left(U^{\prime \prime}{ }_{s} h\right) \\
& =U^{\prime}{ }_{x} W\left(U_{s}^{*} h\right)
\end{aligned}
$$

This shows that $W U_{x}=U_{x}^{\prime} W$ for every $x \in S$, hence for every $x \in G$.

1.3. Twisted semigroup crossed products. Suppose $A$ is a unital $\mathrm{C}^{*}$-algebra and let $\alpha$ be an action of the discrete semigroup $S$ by not necessarily unital endomorphisms of $A$. Let $\lambda$ be a circle-valued multiplier on $S$. A twisted covariant representation of the semigroup dynamical system $(A, S, \alpha)$ with multiplier $\lambda$ is a pair $(\pi, V)$ in which

1. $\pi$ is a unital representation of $A$ on $H$,

2. $V: S \rightarrow \operatorname{Isom}(H)$ is a projective isometric representation of $S$ with multiplier $\lambda$, i.e., $V_{s} V_{t}=\lambda(s, t) V_{s t}$, and

3. the covariance condition $\pi\left(\alpha_{t}(a)\right)=V_{t} \pi(a) V_{t}^{*}$ holds for every $a \in A$ and $t \in S$.

When dealing with twisted covariant representations with a specific multiplier $\lambda$, we will refer to the dynamical system as a twisted dynamical system and denote it by $(A, S, \alpha, \lambda)$.

The (twisted) crossed product associated to $(A, S, \alpha, \lambda)$ is a $\mathrm{C}^{*}$-algebra $A \rtimes_{\alpha, \lambda} S$ together with a unital homomorphism $i_{A}: A \rightarrow A \rtimes_{\alpha, \lambda} S$ and a projective $\lambda$-representation of $S$ as isometries $i_{S}: S \rightarrow A \rtimes_{\alpha, \lambda} S$ such that

1. $\left(i_{A}, i_{S}\right)$ is a twisted covariant representation for $(A, S, \alpha, \lambda)$,

2. for any other covariant representation $(\pi, V)$ there is a representation $\pi \times V$ of $A \rtimes_{\alpha, \lambda} S$ such that $\pi=(\pi \times V) \circ i_{A}$ and $V=(\pi \times V) \circ i_{S}$, and

3. $A \rtimes_{\alpha, \lambda} S$ is generated by $i_{A}(A)$ and $i_{S}(S)$ as a $\mathrm{C}^{*}$-algebra. 
The existence of a nontrivial universal object associated to $(A, S, \alpha, \lambda)$ depends on the existence of a nontrivial twisted covariant representation with multiplier $\lambda$. For general endomorphisms such representations need not exist, even in the untwisted case. For instance, the action of $\mathbb{N}$ by surjective shift-endomorphisms of $c_{0}$ described in Example 2.1(a) of [31] does not admit any nontrivial covariant representations. We will assume that our endomorphisms are injective, hence nontriviality of the semigroup crossed product will follow from its realization as a corner in a nontrivial classical crossed product. See 31, 25] for abelian semigroups, and Remark 2.2.2 below. There are other possible covariance conditions which yield nontrivial crossed products even if the endomorphisms fail to be injective, see e.g. [24] and [28]. We will not deal with them here, but we refer to [22] for an interesting comparative discussion of the different constructions.

Remark 1.3.1. It is immediate from the definition that the crossed product $A \rtimes S$ is generated, as a $\mathrm{C}^{*}$-algebra, by the monomials $v_{x}^{*} a v_{y}$ with $a \in A$, and $x, y \in S$, but more is true for Ore semigroups: the products of such monomials can be simplified using covariance to obtain another monomial of the same type. Specifically, in order to simplify the product $v_{x}^{*} a v_{y} v_{r}^{*} b v_{s}$ we begin by finding elements $t$ and $z$ in $S$ such that $y r^{-1}=t^{-1} z$, so that $t y=z r$, (such elements do exist because $S$ is an Ore semigroup). It follows that

$$
\begin{aligned}
v_{x}^{*} a v_{y} v_{r}^{*} b v_{s} & =\lambda(y, t) \overline{\lambda(z, r)} v_{x}^{*} a v_{y} v_{t y}^{*} v_{z r} v_{r}^{*} b v_{s} \\
& =\lambda(y, t) \overline{\lambda(z, r)} v_{x}^{*} a v_{y} v_{y}^{*} v_{t}^{*} v_{z} v_{r} v_{r}^{*} b v_{s} \\
& =\lambda(y, t) \overline{\lambda(z, r)} v_{x}^{*} v_{t}^{*} \alpha_{t}\left(a \alpha_{y}(1)\right) \alpha_{z}\left(\alpha_{r}(1) b\right) v_{z} v_{s} \\
& =\lambda(y, t) \overline{\lambda(z, r) \lambda(t, x)} \lambda(z, s) v_{t x}^{*} \alpha_{t}\left(a \alpha_{y}(1)\right) \alpha_{z}\left(\alpha_{r}(1) b\right) v_{z s}
\end{aligned}
$$

hence the linear span of such monomials is dense in the crossed product.

\section{The minimal automorphic Dilation.}

There are two steps in realizing a semigroup crossed product as a corner in a crossed product by a group action. The first one is the dilation-extension of a semigroup action by injective endomorphisms to a group action by automorphisms, and the second one is the corresponding dilation-extension of covariant representations of the semigroup dynamical system to covariant representations of the dilated system.

\subsection{A dilation-extension theorem.}

Theorem 2.1.1. Assume $S$ is an Ore semigroup with enveloping group $G=S^{-1} S$ and let $\alpha$ be an action of $S$ by injective endomorphisms of a unital $C^{*}$-algebra $A$. Then there exists a $C^{*}$-dynamical system $(B, G, \beta)$, unique up to isomorphism, consisting of an action $\beta$ of $G$ by automorphisms of a $C^{*}$-algebra $B$ and an embedding $i: A \rightarrow B$ such that

1. $\beta$ dilates $\alpha$, that is, $\beta_{s} \circ i=i \circ \alpha_{s}$ for $s \in S$, and

2. $(B, G, \beta)$ is minimal, that is, $\bigcup_{s \in S} \beta_{s}^{-1}(i(A))$ is dense in $B$.

Proof. By right-reversibility, $S$ is directed by $\preceq_{r}$ so one may follow the argument of 25, Section 2]. However, extra work is needed here: since $G$ need not be abelian, the choice of embeddings in the directed system must be carefully matched to the choice of right-order $\preceq_{r}$ on $S$.

Consider the directed system of $\mathrm{C}^{*}$-algebras determined by the maps $\alpha_{y}^{x}=\alpha_{y x^{-1}}$ from $A_{x}:=A$ into $A_{y}:=A$, for $x \in S$ and $y \in S x$, i.e. for $x \preceq_{r} y$ in $S$. By 15, Proposition 11.4.1(i)] there exists an inductive limit $\mathrm{C}^{*}$-algebra $A_{\infty}$ together with embeddings $\alpha^{x}$ : $A_{x} \rightarrow A_{\infty}$ such that $\alpha^{x}=\alpha^{y} \circ \alpha_{y}^{x}$ whenever $x \preceq_{r} y$, and such that $\bigcup_{x \in S} \alpha^{x}\left(A_{x}\right)$ is dense in $A_{\infty}$. 
The next step is to extend the endomorphism $\alpha_{s}$ to an automorphism of $A_{\infty}$. For any fixed $s \in S$ the subset $S s$ of $S$ is cofinal, so $A_{\infty}$ is also the inductive limit of the directed subsystem $\left(A_{x}, x \in S s\right)$, and, for this subsystem, we may consider new embeddings $\psi^{x}$ : $A_{x} \rightarrow A_{\infty}$ defined by $\psi^{x}(a)=\alpha^{x s^{-1}}(a)$ for $x \in S s$ and $a \in A_{x}$. By 15, Proposition 11.4.1(ii)] there is an automorphism $\tilde{\alpha}_{s}$ of $A_{\infty}$ such that $\tilde{\alpha}_{s} \circ \alpha^{x}=\psi^{x}$ for every $x \in S s$. Since $\alpha^{1}=\alpha^{s} \circ \alpha_{s}^{1}$ and $\psi^{x}=\alpha^{x s^{-1}}$, the choice $x=s$ gives

$$
\tilde{\alpha}_{s} \circ \alpha^{1}=\tilde{\alpha}_{s} \circ \alpha^{s} \circ \alpha_{s}^{1}=\alpha^{1} \circ \alpha_{s}
$$

so that (1) holds with $\beta=\tilde{\alpha}$ and $i=\alpha^{1}: A_{1} \rightarrow A_{\infty}$.

Since $\tilde{\alpha}_{s}^{-1}(i(A))=\alpha^{s}\left(A_{s}\right),(2)$ also holds. Uniqueness of the dilated system follows from [15, Proposition 11.4.1(ii)]: $A_{\infty}$ is the closure of the union of the subalgebras $\tilde{\alpha}_{s}^{-1}(i(A))$ with $s \in S$, if $(B, G, \beta)$ is another minimal dilation with embedding $j: A \rightarrow B$ then there is an isomorphism $\theta: A_{\infty} \rightarrow B$ given by $\theta \circ \tilde{\alpha}_{s^{-1}}(i(a))=\beta_{s^{-1}}(j(a))$ for $a \in A$ and hence which intertwines $\tilde{\alpha}$ and $\beta$.

Definition 2.1.2. A system $(B, G, \beta)$ satisfying the conditions (1) and (2) of Theorem 2.1.1 is called the minimal automorphic dilation of $(A, S, \alpha)$. If $\lambda$ is a multiplier on $S$ with extension $\mu$ to $G$, we say that the twisted system $(B, G, \beta, \mu)$ is the minimal automorphic dilation of the twisted system $(A, S, \alpha, \lambda)$. (By Theorem 1.2 .2 the extended multiplier exists and is unique up to a coboundary.)

Lemma 2.1.3. Let $(\pi, V)$ be a covariant representation for the twisted system $(A, S, \alpha, \lambda)$ on the Hilbert space $H$, and let $\tilde{V}$ be the minimal projective unitary dilation of $V$ on $\mathcal{H}$ given by Theorem 1.2.1. Then there exists a representation $\tilde{\pi}$ of $B$ on $\mathcal{H}$ such that $(\tilde{\pi}, \tilde{V})$ is covariant for the minimal automorphic dilation $(B, G, \beta, \mu)$ and $\tilde{\pi} \circ i=\pi$ on $H$.

Proof. We work with the dense subspace $\mathcal{H}_{0}=\bigcup_{t \in S} U_{t}^{*} H$ of $\mathcal{H}$ and the dense subalgebra $B_{0}=\bigcup_{s \in S} \beta_{s}^{-1}(i(A))$. If $\xi \in \mathcal{H}_{0}$ there exists $t \in S$ such that $U_{t} \xi \in H$; assume $b=$ $\beta_{t}^{-1}(i(a))$, since we want $(\tilde{\pi}, \tilde{V})$ to be covariant, the only choice is to define $\tilde{\pi}$ by

$$
\tilde{\pi}(b) \xi=\tilde{\pi}\left(\beta_{t}^{-1}(i(a))\right) \xi=\tilde{V}_{t}^{*} \tilde{\pi}(i(a)) \tilde{V}_{t} \xi=\tilde{V}_{t}^{*} \pi(a) \tilde{V}_{t} \xi
$$

because $\tilde{\pi}$ restricted to $i(A)$ and cut down to $H$ has to be equal to $\pi$.

Of course we have to show that this actually defines an operator $\tilde{\pi}(b)$ on $\mathcal{H}$ for each $b \in B_{0}$, that $\tilde{\pi}$ extends to a homomorphism from all of $B$ to $B(\mathcal{H})$, and that $(\tilde{\pi}, \tilde{V})$ is covariant.

The first step is to define $\tilde{\pi}(b)$ on $\mathcal{H}_{0}$ for a fixed $b \in B_{0}$. We begin by fixing $b \in B_{0}$, $a \in A$ and $s \in S$ such that $b=\beta_{s}^{-1}(i(a))$. For $\xi \in \tilde{V}_{t_{0}}^{*} H$ with $t_{0}$ in the cofinal set $S s$, we let

$$
\varphi(b) \xi=\tilde{V}_{t_{0}}^{*} \pi\left(\alpha_{t_{0} s^{-1}}(a)\right) \tilde{V}_{t_{0}} \xi .
$$

If $t \in S t_{0}$ then $\xi \in \tilde{V}_{t}^{*} H$, and

$$
\begin{aligned}
\tilde{V}_{t}^{*} \pi\left(\alpha_{t s^{-1}}(a)\right) \tilde{V}_{t} \xi_{0} & =\tilde{V}_{t_{0}}^{*} \tilde{V}_{t t_{0}^{-1}}^{*} \pi\left(\alpha_{t t_{0}^{-1}} \circ \alpha_{t_{0} s^{-1}}(a)\right) \tilde{V}_{t t_{0}^{-1}} \tilde{V}_{t_{0}} \xi \\
& =\tilde{V}_{t_{0}}^{*} \tilde{V}_{t t_{0}^{-1}}^{*} V_{t t_{0}^{-1}} \pi\left(\alpha_{t_{0} s^{-1}}(a)\right) V_{t t_{0}^{-1}}^{*} \tilde{V}_{t t_{0}^{-1}} \tilde{V}_{t_{0}} \xi \\
& =\tilde{V}_{t_{0}}^{*} \pi\left(\alpha_{t_{0} s^{-1}}(a)\right) \tilde{V}_{t_{0}} \xi
\end{aligned}
$$

So the definition of $\phi(b) \xi$ could have been given using any $t \in S t_{0}$ in place of $t_{0}$. Next we show that $\phi(b) \xi$ is also independent of $s$ and $a$, in the sense that if $b$ is also equal to $\beta_{s^{\prime}}^{-1}\left(i\left(a^{\prime}\right)\right)$ then $\alpha_{t s^{\prime}}\left(a^{\prime}\right)$ is equal to $\alpha_{t s^{-1}}(a)$ for $t$ in a cofinal set. To see this let 
$t \in S s \cap S s^{\prime}$. Then $\alpha^{t} \circ \alpha_{t}^{s^{\prime}}\left(a^{\prime}\right)=\alpha^{s^{\prime}}\left(a^{\prime}\right)=\beta_{s^{\prime}}^{-1}\left(i\left(a^{\prime}\right)\right)=\beta_{s}^{-1}(i(a))=\alpha^{s}(a)=\alpha^{t} \circ \alpha_{t}^{s}(a)$, and since the embedding $\alpha^{t}$ is injective, it follows that $\alpha_{t s^{\prime-1}}\left(a^{\prime}\right)=\alpha_{t s^{-1}}(a)$.

The map $\varphi(b): \mathcal{H}_{0} \rightarrow \mathcal{H}_{0}$ is clearly linear, and since the endomorphisms are injective, $\|\varphi(b) \xi\| \leq\|b\|\|\xi\|$. Thus $\phi(b)$ can be uniquely extended to a bounded linear operator (also denoted $\varphi(b))$ on all of $\mathcal{H}$ such that $\|\varphi(b)\| \leq\|b\|$. For any $s$ the map $\operatorname{Ad}_{\tilde{V}_{t_{0}}^{*}} \circ \pi \circ \alpha_{t_{0} s^{-1}}$ is a *-homomorphism on $A$, and by cofinality of $\preceq_{r}$, for any $b_{1}$ and $b_{2}$ in $B_{0}$ there exist $s \in S$ and $a_{1}$ and $a_{2}$ in $A$ such that $b_{1}=\beta_{s}^{-1}\left(i\left(a_{1}\right)\right)$ and $b_{2}=\beta_{s}^{-1}\left(i\left(a_{2}\right)\right)$. It follows easily from (2.1.1) that $\varphi: B_{0} \rightarrow B(\mathcal{H})$ is a ${ }^{*}$-homomorphism which can be extended to a representation $\tilde{\pi}$ of $B$ on $\mathcal{H}$.

Putting $a=1$ in (2.1.1) shows that $\tilde{\pi}$ is nondegenerate and there only remains to check that $(\tilde{\pi}, \tilde{V})$ is a covariant pair for $(B, G, \beta, \mu)$.

Suppose first $x \in S$ and $b \in B_{0}$; we can assume that $b=\beta_{s}^{-1}(i(a))$ for some $a \in A$ and $s \in S x$. Let $\xi \in \tilde{V}_{t}^{*} H$; we can assume $t \in S s \subset S x$, and we observe that $\tilde{V}_{x} \xi \in \tilde{V}_{t x^{-1}}^{*} H$. Then

$$
\begin{aligned}
\tilde{\pi}\left(\beta_{x}(b)\right) \tilde{V}_{x} \xi & =\tilde{\pi}\left(\beta_{x s^{-1}}(i(a))\right) \tilde{V}_{x} \xi \\
& =\tilde{\pi}\left(\beta_{s x^{-1}}^{-1}(i(a))\right) \tilde{V}_{x} \xi \\
& =\tilde{V}_{t x^{-1}}^{*} \pi\left(\alpha_{t x^{-1} x s^{-1}}(i(a))\right) \tilde{V}_{t x^{-1}} \tilde{V}_{x} \xi \\
& =\tilde{V}_{t x^{-1}}^{*} \pi\left(\alpha_{t s^{-1}}(i(a))\right) \tilde{V}_{t x^{-1}} \tilde{V}_{x} \xi \\
& =\tilde{V}_{x^{-1}}^{*} \tilde{V}_{t}^{*} \pi\left(\alpha_{t s^{-1}}(i(a))\right) \tilde{V}_{t} \xi \\
& =\tilde{V}_{x} \tilde{\pi}\left(\beta_{s}^{-1}(i(a))\right) \xi
\end{aligned}
$$

and since $\mathcal{H}_{0}$ is dense in $\mathcal{H}$ and $B_{0}$ is dense in $B$, the pair $(\tilde{\pi}, \tilde{V})$ satisfies the covariance relation.

2.2. Full corners. Once we know how to dilate covariant representations from the semigroup action to the group action we can establish the relation between the respective crossed products. Before proving our main result we recall that if $p$ is a projection in the $\mathrm{C}^{*}$-algebra $A$ then the algebra $p A p$ is a corner in $A$, which is said to be full if the linear span of $A p A$ is dense in $A$. The most relevant feature of full corners is that if $p A p$ is a full corner in $A$, then $p A$ is a full Hilbert bimodule implementing the Morita equivalence, in the sense of Rieffel, of $p A p$ to $A$.

Theorem 2.2.1. Suppose $(A, S, \alpha, \lambda)$ is a twisted semigroup dynamical system in which $S$ is an Ore semigroup acting by injective endomorphisms and $\lambda$ is a multiplier on $S$. Let $(B, G, \beta, \mu)$ be the minimal automorphic dilation, with embedding $i: A \rightarrow B$. Then $A \rtimes_{\alpha, \lambda} S$ is canonically isomorphic to $i(1)\left(B \rtimes_{\beta, \mu} G\right) i(1)$, which is a full corner. As a consequence, the crossed product $A \rtimes_{\alpha, \lambda} S$ is Morita equivalent to $B \rtimes_{\beta, \mu} G$.

Proof. Let $U$ be the projective unitary representation of $G$ in the multiplier algebra of $B \rtimes_{\beta, \mu} G$, and notice that

$$
i(1) U_{s} i(1)=U_{s} i(1), \quad s \in S,
$$

because $i(A)$ is invariant under $\beta_{s}$. Define $v_{s}=U_{s} i(1)$. Then $v_{s}^{*} v_{s}=i(1) U_{s}^{*} U_{s} i(1)=i(1)$ and $v_{s} v_{t}=U_{s} i(1) U_{t} i(1)=U_{s} U_{t} i(1)=\mu(s, t) U_{s t} i(1)=\lambda(s, t) v_{s t}$, so $v$ is a projective isometric representation of $S$ with multiplier $\lambda$. Since $i(1)\left(B \rtimes_{\beta, \mu} G\right) i(1)$ is generated by the elements $i(1) U_{x}^{*} i(a) U_{y} i(1)=v_{x}^{*} i(a) v_{y}$, the isomorphism will be established by uniqueness of the crossed product once we show that the pair $(i, v)$ is universal.

Suppose $(\pi, V)$ is a covariant representation for the twisted system $(A, S, \alpha, \lambda)$, and let $(\tilde{\pi}, \tilde{V})$ be the corresponding dilated covariant representation of $(B, G, \beta, \mu)$ given by 
Lemma 2.1.3. By the universal property of $B \rtimes_{\beta, \mu} G$ there is a homomorphism

$$
(\tilde{\pi} \times \tilde{V}): B \rtimes_{\beta, \mu} G \rightarrow C^{*}(\tilde{\pi}, \tilde{V})
$$

such that $\tilde{\pi}(b) \tilde{V}_{s}=(\tilde{\pi} \times \tilde{V})\left(i_{B}(b) U_{s}\right)$.

Let $\rho$ be the restriction of $(\tilde{\pi} \times \tilde{V})$ to $i(1)\left(B \rtimes_{\beta, \mu} G\right) i(1)$, cut down to the invariant subspace $H$. By Lemma 2.1.3

$$
\rho(i(a))=(\tilde{\pi} \times \tilde{V})(i(a))=\tilde{\pi} \circ i(a)=\pi(a), \quad a \in A,
$$

while

$$
\rho\left(v_{s}\right)=(\tilde{\pi} \times \tilde{V})\left(U_{s} i(1)\right)=\tilde{V}_{s} \pi(1)=V_{s}, \quad s \in S
$$

Thus $\rho \circ i=\pi$ and $\rho \circ v=V$, so $(i, v)$ is universal for $(A, S, \alpha, \lambda)$.

Finally we prove that the corner is full, i.e., that the linear span of the elements of the form $X i(1) Y$ with $X, Y \in B \rtimes_{\beta, \mu} G$ is a dense subset of $B \rtimes_{\beta, \mu} G$. It is easy to see that the elements of the form $U_{s}^{*} b U_{t}$ span a dense subset of $B \rtimes_{\beta, \mu} G$ because $G=S^{-1} S$, where $b$ may be replaced with $U_{r}^{*} i(a) U_{r}$ by minimality of the dilation. Thus the elements $U_{y}^{*} i\left(\alpha_{z}(a)\right) U_{x}$ with $x, y, z \in S$ and $a \in A$ span a dense subset of $B \rtimes_{\beta, \mu} G$, and since $i\left(\alpha_{z}(a)\right)=i(1) i\left(\alpha_{z}(a)\right)$, the proof is finished.

Remark 2.2.2. If one drops the assumption of injectivity of the endomorphisms, it is still possible to carry out the constructions and the arguments in the proofs of the preceding theorems. However, the resulting homomorphism $i: A \rightarrow B$ may not be an embedding any more. Indeed, Example 2.1(a) of [31] shows that the limit algebra $B$ may turn out to be the $0 \mathrm{C}^{*}$-algebra, yielding a trivial dilated system.

We notice that the dilated system $(B, G, \beta, \mu)$ has nontrivial covariant representations if and only if $B \neq 0$, and these representations, when cut down to $i(A)$, give nontrivial covariant representations of the original semigroup system $(A, S, \alpha, \lambda)$. Thus, following 31 , Proposition 2.2] which deals with the case $S=\mathbb{N}$, we conclude that the crossed product $A \rtimes_{\alpha, \lambda} S$ is nontrivial if and only if the limit algebra $B$ is not 0 . Clearly, this is the case when, for instance, the endomorphisms are injective.

\section{An EXAMPLE FROM NUMBER THEORY.}

As an application of the preceding theory we consider the semigroup dynamical system from [21] whose crossed product is the Bost-Connes Hecke $\mathrm{C}^{*}$-algebra [5]. Since Morita equivalence implies that the representation theory of the semigroup dynamical system is equivalent to that of the dilated system, it is quite useful to have an explicit formulation of the dilation. We point out that since the semigroup in question is abelian, this application is somewhat independent from the rest of the material on nonabelian semigroups. In fact, the example could be dealt with by enhancing [25, Section 2] with the uniqueness and fullness properties discussed above, which are easier to prove for abelian semigroups.

3.1. Finite Adeles. The natural setting for identifying the ingredients of the minimal automorphic dilation of the semigroup dynamical system introduced in 21] will be the (dual) $p$-adic picture described in [18, Proposition 32], in which the algebra is $C\left(\prod_{p} \mathbb{Z}_{p}\right)$ and the endomorphisms $\alpha_{n}$ consist of 'division by $n$ ' in $\prod_{p} \mathbb{Z}_{p}$ :

$$
\alpha_{n}(f)(x)= \begin{cases}f(x / n) & \text { if } n \mid x \\ 0 & \text { otherwise. }\end{cases}
$$

By [21, Corollary 2.10] the crossed product associated to this system is canonically isomorphic to the Bost-Connes Hecke $\mathrm{C}^{*}$-algebra $\mathcal{C}_{\mathbb{Q}}$. 
The ring $\mathcal{Z}:=\prod_{p} \mathbb{Z}_{p}$ has lots of zero divisors and hence no fraction field. However, the diagonally embedded copy of $\mathbb{N}^{\times}$is a multiplicative set with no zero divisors, and we may enlarge $\mathcal{Z}$ to a ring in which division by an element of $\mathbb{N}^{x}$ is always possible. Our motivation is to extend the endomorphisms $\alpha_{n}$ defined above to automorphisms.

The algebraic part is easy: we consider the ring $\left(\mathbb{N}^{\times}\right)^{-1} \mathcal{Z}$ of formal fractions $z / n$ with $z \in \mathcal{Z}$ and $n \in \mathbb{N}^{\times}$, with the obvious rules of addition and multiplication (and simplification!), [23, II.§3]. This ring has a universal property with respect to homomorphisms of $\mathcal{Z}$ that send $\mathbb{N}^{\times}$into units. Since $\mathbb{N}^{\times}$has no zero divisors, the canonical map $z \rightarrow z / 1$ is an embedding of $\mathcal{Z}$ in $\left(\mathbb{N}^{\times}\right)^{-1} \mathcal{Z}$.

The topological aspect requires a moments thought, after which we declare that the subring $\mathcal{Z}$ must retain its compact topology and be relatively open. Since we want division by $n \in \mathbb{N}^{\times}$to be an automorphism, this determines a topology on the compact open sets $(1 / n) \mathcal{Z}$ and hence on their union, $\left(\mathbb{N}^{\times}\right)^{-1} \mathcal{Z}$, which becomes a locally compact ring containing $\mathcal{Z}$ as a compact open subring.

The ring we have just defined is (isomorphic to) the locally compact ring $\mathbb{A}_{f}$ of finite adeles, which is usually defined as the restricted product, over the primes $p \in \mathcal{P}$ of the $p$-adic numbers $\mathbb{Q}_{p}$ with respect to the $p$-adic integers $\mathbb{Z}_{p}$ :

$$
\mathbb{A}_{f}:=\left\{\left(a_{p}\right): a_{p} \in \mathbb{Q}_{p} \text { and } a_{p} \in \mathbb{Z}_{p} \text { for all but finitely many } p \in \mathcal{P}\right\},
$$

with $\prod_{p} \mathbb{Z}_{p}$ as its maximal compact open subring. The isomorphism is implemented by the map from $\left(\mathbb{N}^{\times}\right)^{-1} \mathcal{Z}$ into $\mathbb{A}_{f}$ given by the universal property; this map is clearly injective and, since every finite adele can be written as $z / n$ with $z \in \mathcal{Z}$ and $n \in \mathbb{N}^{\times}$, it is also surjective. Specifically, for each $a_{p} \in \mathbb{Q}_{p}$ there exists $k_{p}$ such that $p^{k_{p}} a_{p}=z_{p} \in \mathbb{Z}_{p}$ and a sequence $a=\left(a_{p}\right)_{p \in \mathcal{P}}$ is an adele if and only if $k_{p}$ can be taken to be 0 for all but finitely many $p$ 's, in which case $n=\prod_{p} p^{-k_{p}} \in \mathbb{N}^{\times}$and $a=(n a) / n$, with $n a=\left(n a_{p}\right)_{p \in \mathcal{P}} \in \prod_{p} \mathbb{Z}_{p}$.

3.2. The minimal automorphic dilation of $\left(C(\mathcal{Z}), \mathbb{N}^{\times}, \alpha\right)$. The rational numbers are embedded in $\mathbb{A}_{f}$, and division by a nonzero rational is clearly a homeomorphism so

$$
\beta_{r}(f)(a)=f\left(r^{-1} a\right), \quad a \in \mathbb{A}_{f}, r \in \mathbb{Q}_{+}^{*}
$$

defines an action of $\mathbb{Q}_{+}^{*}=\left(\mathbb{N}^{\times}\right)^{-1} \mathbb{N}^{\times}$by automorphisms of $C_{0}\left(\mathbb{A}_{f}\right)$.

Since $\mathcal{Z}$ is compact and open, its characteristic function $1_{\mathcal{Z}}$ is a projection in $C_{0}\left(\mathbb{A}_{f}\right)$ and there is an obvious embedding $i$ of $C(\mathcal{Z})$ as the corresponding ideal of $C_{0}\left(\mathbb{A}_{f}\right)$, given by

$$
i(f)(a)= \begin{cases}f(a) & \text { if } a \in \mathcal{Z} \\ 0 & \text { if } a \notin \mathcal{Z}\end{cases}
$$

Proposition 3.2.1. The $C^{*}$-dynamical system $\left(C_{0}\left(\mathbb{A}_{f}\right), \mathbb{Q}_{+}^{*}, \beta\right)$ is the minimal automorphic dilation of the semigroup dynamical system $\left(C(\mathcal{Z}), \mathbb{N}^{\times}, \alpha\right)$, and hence $\mathcal{C}_{\mathbb{Q}}$ is the full corner of $C_{0}\left(\mathbb{A}_{f}\right) \rtimes_{\beta} \mathbb{Q}_{+}^{*}$ determined by the projection $1_{\mathcal{Z}}$.

Proof. The embedding clearly intertwines $\alpha_{n}$ and $\beta_{n}$, in the sense that $\beta_{n}(i(f))=i\left(\alpha_{n}(f)\right)$, and the union of the compact subgroups $(1 / n) \mathcal{Z}$ is dense in $\mathbb{A}_{f}$, so the union of the subalgebras $\beta_{1 / n}(i(C(\mathcal{Z})))$ is dense in $C_{0}\left(\mathbb{A}_{f}\right)$, and the result follows from Theorem 2.1.1 and Theorem 2.2.1.

Since the discrete multiplicative group $\mathbb{Q}_{+}^{*}$ acts by homotheties on the locally compact additive group $\mathbb{A}_{f}$, and since $\mathbb{A}_{f}$ is self-dual, we obtain another characterization of $\mathcal{C}_{\mathbb{Q}}$ as a full corner in the group $C^{*}$-algebra of the semidirect product $\mathbb{A}_{f} \rtimes \mathbb{Q}_{+}^{*}$. One should bear in mind, however, that the self duality of $\mathbb{A}_{f}$ is not canonical. 
Corollary 3.2.2. Let $e_{\mathcal{Z}} \in C^{*}\left(\mathbb{A}_{f}\right)$ be the Fourier transform of $1_{\mathcal{Z}} \in C_{0}\left(\mathbb{A}_{f}\right)$. Then

$$
\mathcal{C}_{\mathbb{Q}} \cong e_{\mathcal{Z}} C^{*}\left(\mathbb{A}_{f} \rtimes \mathbb{Q}_{+}^{*}\right) e_{\mathcal{Z}}
$$

Proof. The action of $\mathbb{Q}_{+}^{*}$ on $\mathbb{A}_{f}$ is by homotheties, which are group automorphisms, so $C^{*}\left(\mathbb{A}_{f} \rtimes \mathbb{Q}_{+}^{*}\right)$ is isomorphic to the crossed product $C^{*}\left(\mathbb{A}_{f}\right) \rtimes_{\beta} \mathbb{Q}_{+}^{*}$. Moreover, the selfduality of the additive group of $\mathbb{A}_{f}$ satisfies $\langle r x, y\rangle=\langle x, r y\rangle$ for $r \in \mathbb{Q}_{+}^{*}$, thus $C^{*}\left(\mathbb{A}_{f}\right)$ is covariantly isomorphic to $C_{0}\left(\mathbb{A}_{f}\right)$, so $C^{*}\left(\mathbb{A}_{f}\right) \rtimes_{\beta} \mathbb{Q}_{+}^{*}$ is isomorphic to $C_{0}\left(\mathbb{A}_{f}\right) \rtimes_{\beta} \mathbb{Q}_{+}^{*}$, and the claim follows from Proposition 3.2.1.

Remark 3.2.3. One of the principles of noncommutative geometry advocates that if $G$ is a group acting on a space $X$, then the quotient space $X / G$ has a noncommutative version in the associated crossed product $C_{0}(X) \rtimes G$, which is often more tractable. Accordingly, if we allow back in the all-important place at infinity which is left out from $\mathcal{A}_{f}$ and if we substitute $\mathbb{Q}_{+}^{*}$ by $\mathbb{Q}^{*}$, cf. $[5$, Remarks 33], then our Proposition 3.2.1 gives an explicit path leading from the Bost-Connes Hecke $\mathrm{C}^{*}$-algebra to the space $\mathcal{A} / \mathbb{Q}^{*}$, on which the construction of [9, 10] is based.

\section{REFERENCES}

[1] S. Adji, PhD Thesis, The University of Newcastle (1995).

[2] S. Adji, M. Laca, M. Nilsen and I. Raeburn, Crossed products by semigroups of endomorphisms and the Toeplitz algebras of ordered groups, Proc. Amer. Math. Soc. 122 (1994), 1133-1141.

[3] J. Arledge, M. Laca and I. Raeburn, Semigroup crossed products and Hecke algebras arising from number fields, Doc. Math. 2 (1997), 115-138.

[4] W. B. Arveson, An addition formula for the index of semigroups of endomorphisms of $\mathcal{B}(\mathcal{H})$, Pacific J. Math. 137 (1989), 19-36.

[5] J.-B. Bost and A. Connes, Hecke algebras, Type III factors and phase transitions with spontaneous symmetry breaking in number theory, Selecta Math. (New Series) 1 (1995), 411-457.

[6] B. Brenken, Hecke algebras and semigroup crossed product $\mathrm{C}^{*}$-algebras, University of Calgary, preprint (1996).

[7] A. H. Clifford and G. B. Preston, "The Algebraic Theory of Semigroups," vol I, Mathematical Surveys, No. 7, Amer. Math. Soc., Providence, RI, 1961.

[8] P. R. Chernoff, Extensions and triviality of multipliers on subsemigroups of the reals. Semigroup Forum 41 (1990), 237-244.

[9] A. Connes, Formule de trace en géométrie non commutative et hypothèse de Riemann, C. R. Acad. Sci. Paris t.323, Série 1 (Analyse), (1996) 1231-1236.

[10] A. Connes, Trace formula in noncommutative Geometry and the zeros of the Riemann zeta function, I.H.E.S. preprint (1997).

[11] J. Cuntz, Simple $C^{*}$-algebras generated by isometries, Comm. Math. Phys. 57 (1977), 173-185.

[12] H. Dinh, Multipliers on subsemigroups of the real line. Proc. Amer. Math. Soc. 117 (1993), $783-788$.

[13] R. G. Douglas, On extending commutative semigroups of isometries. Bull. London Math. Soc. 1 (1969), 157-159.

[14] T. Ito, On the commutative family of subnormal operators, J. Fac. Sci. Hokkaido Univer. 14 (1958), $1-15$.

[15] R. Kadison and J. Ringrose, "Fundamentals of the Theory of Operator Algebras" vol. II, Academic Press, New York.

[16] A. Kleppner, Multipliers on subsemigroups of locally compact abelian groups, Semigroup Forum 47 (1993), 81-86.

[17] M. Laca Discrete product systems with twisted units, Bull. Austral. Math. Soc. 52 (1995), 317-326.

[18] M. Laca, Semigroups of *-endomorphisms, Dirichlet series and phase transitions, J. Funct. Anal. 152 (1998), 330-378.

[19] M. Laca and I. Raeburn, Extending multipliers from semigroups, Proc. Amer. Math. Soc. 123 (1995), 355-362.

[20] M. Laca and I. Raeburn, Semigroup crossed products and the Toeplitz algebras of nonabelian groups, J. Funct. Anal., 139 (1996), 415-440.

[21] M. Laca and I. Raeburn, A semigroup crossed product arising in number theory, J. London Math. Soc., to appear. 
[22] M. Lamoureux, Covariant conditions for crossed product algebras, University of Calgary, preprint (1998).

[23] S. Lang, "Algebra", Academic Press, Reading, Massachussets, 1965.

[24] G. J. Murphy, Ordered groups and crossed products of $C^{*}$-algebras, Pacific J. Math. 148 (1991), 319-349.

[25] G. J. Murphy, Crossed products of $C^{*}$-algebras by endomorphisms, Integr. Equat. Oper. Th. 24 (1995), 298-319.

[26] G. J. Murphy, Extensions of multipliers and dilations of projective isometric representations, Proc. Amer. Math. Soc. 125 (1997), 121-127

[27] W. Paschke, The crossed product of a $\mathrm{C}^{*}$-algebra by an endomorphism, Proc. Amer. Math. Soc. 80 (1980), 113-118.

[28] J. Peters, Semicrossed products of C*-algebras, J. Funct. Anal. 59 (1984), 498-534.

[29] J. Phillips and I. Raeburn, Semigroups of isometries, Toeplitz algebras and twisted crossed products, Integr. Equat. Oper. Th. 17 (1993), 579-602.

[30] I. Raeburn, On crossed products and Takai duality, Proc. Edin. Math. Soc. 31 (1988), 321-330.

[31] P. J. Stacey, Crossed products of $\mathrm{C}^{*}$-algebras by *-endomorphisms, J. Austral. Math. Soc., (Series A) 54 (1993), 204-212.

Department of Mathematics, The University of Newcastle, NSW 2308, AUSTRALiA

E-mail address: marcelo@math.newcastle.edu.au 